\title{
III. Arbeitsvorhaben und Delegationen der Akademie
}

\author{
Papsturkunden- und mittelalterliche Geschichtsforschung (Pius-Stiftung) \\ Wissenschaftliche Kommission: \\ Vorsitzender: der Vorsitzende der Phil.-Hist. Klasse \\ Sekretär: Herbers (Erlangen) \\ Maleczek (Wien), Paravicini-Bagliani (Lausanne), Pasini (Città del Vati- \\ cano), Schieffer
}

Kontaktadresse: Friedländer Weg 11, 37085 Göttingen, Tel.: 05515316499, Fax: 0551-5316512,

wkoenig@gwdg.de (Dr. Könighaus), http://www.papsturkunden.gwdg.de

Arbeitsbericht: Frau Andrea Birnstiel (Göttingen) hat in der Göttinger Arbeitsstelle die Bearbeitung der Sammlung „Papsturkunden aus Drucken“ fortgesetzt, im einzelnen die Durchsicht des Materials, Aufnahme weiterer Stücke und Aktualisierung der zugehörigen Datenbank „Papsturkunden Anfänge bis 1198“. Die Sammlung enthält derzeit Materialien zu 7.948 Papsturkunden (Stand: 30. September 2010). Weiterhin konnte die Photosammlung der Arbeitsstelle in die im Dezember 2009 erworbenen Stahlschränke transferiert und auf diese Weise archiviert werden. Im Zuge dessen wurde sie in einer neuen Datenbank („Photosammlung“) erfaßt. Sowohl in der Datenbank als auch in der Sammlung selbst wird dabei auch auf Materialien in anderen Sammlungen der Arbeitsstelle verwiesen. Parallel dazu konnte diese Sammlung durch neue Stücke ergänzt werden.

Im Dezember 2009 hielten sich Herr Dr. Joachim Dahlhaus (Eppelheim), im Juni 2010 Frau Claudia Alraum, M. A. und Herr Andreas Holndonner (beide Erlangen) und im Oktober 2010 Herr Prof. Dr. Rudolf Hiestand (Düsseldorf) zu Forschungszwecken in der Arbeitsstelle auf.

Italia Pontificia

Ein Bericht von Prof. Dr. Raffaello Volpini (Rom) lag nicht vor.

Germania Pontificia

Bd. VIII (Diözese Lüttich): Die Arbeiten von Herrn Dr. Wolfgang Peters (Köln) konzentrierten sich im Berichtszeitraum auf die Abtei Stablo-Malmedy. Nach einer Phase der Einarbeitung stellte sich heraus, daß zu den Papsturkunden des 10. und des 11. Jahrhunderts Einzelstudien notwendig sind, bevor diese Privilegien in Regestenform gebracht werden können. 
Diese Voruntersuchungen hofft der Autor in diesem und im nächsten Jahr abschließen zu können. Gedacht ist an kleinere Beiträge, die in den einschlägigen Zeitschriften veröffentlicht werden sollen. - Bd. XI (Diözese Toul): Herr Dr. Joachim Dahlhaus (Eppelheim) übernahm im Berichtszeitraum die Bearbeitung der Diözese Toul. Seine bisherige Tätigkeit bestand hauptsächlich in der Prüfung der ihm vom Sekretär anvertrauten Materialien. Außerdem bemühte er sich um die Ermittlung und Beschaffung ergänzender Quellen und einschlägiger Literatur. - Bd. XII (Erzdiözese Magdeburg): Ein Bericht von Herrn Dr. Jürgen Simon (Hamburg) lag nicht vor. Bd. XIII (Regnum und Imperium): Herr Prof. Dr. Hans H. Kaminsky (Gießen) setzte seine Arbeiten am bibliographischen Apparat fort. - Bd. XIV (Supplementum I): Seit dem Frühjahr 2010 galt Herrn Hiestands Arbeit der Fertigstellung dieses Bandes, und zwar der Erstellung der Narrationes für die ca. 50 neuen Lemmata. Dabei war die bibliographische Recherche und dann vor allem die Beschaffung der ermittelten Literatur nicht nur zeitaufwendig, sondern manchmal auch erfolglos. Der Großteil der Ergänzungen betrifft die Bände „Germania Pontificia I und III“. - In die Indices zur „Germania“, die für die bereits veröffentlichten Bände schon fertiggestellt sind, wird Herr Hiestand noch den Band Böhmen einarbeiten, sobald die Numerierung der Stücke und die Seitenzahlen endgültig feststehen.

\section{Gallia Pontificia}

Nachdem Prof. Dr. Dietrich Lohrmann (Aachen) die Leitung der „Gallia Pontificia" niedergelegt hat, wird diese zur Zeit vom Sekretär kommissarisch versehen.

1. Diözesen Reims und Châlons: Der Altsekretär hat das Manuskript von Dr. Ludwig Falkenstein (Aachen) für die Erzbischöfe von Reims durchgesehen. Der Band soll in der Reihe „Studien und Dokumente zur Gallia Pontificia" erscheinen und wird nach der Einarbeitung der Korrekturen durch Herrn Falkenstein von Herrn Prof. Dr. Rolf Große (Paris/Heidelberg) zum Druck vorbereitet. - 2. Diözese Paris: Im Berichtszeitraum nahm Herr Große für den zehnten Band der „Papsturkunden in Frankreich“ Ergänzungen des Editionsteils und des Archivberichts vor. Ferner leitete er die Drucklegung von Bd. 7 der "Studien und Dokumente zur Gallia Pontificia“ in die Wege und unternahm erste Schritte zur Organisation der nächsten Table ronde. Des weiteren führte er einen Jahrgang der École des chartes in das Projekt ein und stellte es fortgeschrittenen Studenten der Universität Paris 8 vor. Zur Neustrukturierung des Projekts und der Einrichtung einer digitalen Plattform führte er mehrere Gespräche. -3 . Diözese Langres 
(Prof. Benoît Chauvin, Devecey): Ein Bericht lag nicht vor. - 4. Diözese Thérouanne, Abtei Saint-Bertin (Prof. Laurent Morelle, Paris): Ein Bericht lag nicht vor. - I/1: Erzdiözese Besançon: Der Band liegt vor (1998). I/2: Suffragane: P. Bernard de Vregille (Lyon) gab mit Blick auf sein hohes Alter die von ihm bearbeiteten Abschnitte des Bandes zurück und verwies darauf, daß seine Manuskripte für das Bistum Belley und den Abschnitt „Besançon-Supplément“ schon seitlängerer Zeit fertig sind. Zu den Bistümern Lausanne (Prof. Jean-Daniel Morerod, Neuchâtel) und Basel (Archivdirektoren Jean-Luc Eichenlaub, Colmar, und Jean-Claude Rebetez, Porrentruy/Pruntrut) ist jeweils kein Bericht eingegangen. - II/1: Erzdiözese Lyon (Prof. Michel Rubellin/Prof. Denyse Riche): Ein Bericht lag nicht vor. - II/2: Suffragane Lyon, insbesondere Diözese Mâcon mit der Abtei Cluny (Dr. Franz Neiske, Münster): Der Bearbeiter wies in seinem Bericht auf die online abrufbaren Urkunden der Abtei (Cartae Cluniacenses Electronicae) hin. Auch Herr Gérard Moyse (Dijon) konnte keine weiteren Arbeitsfortschritte für den Regestenband vermelden. - III/1: Erzdiözese Vienne: Der Band liegt vor (2006). - III/2: Suffragane Vienne: Frau Dr. Beate Schilling (München) befaßte sich im Berichtsjahr überwiegend mit der Abfassung von Aufsätzen (unter anderem einem Tagungsbeitrag zu den Kartäusern für die letzte Table ronde). Außerdem widmete sie sich den ca. 50 Regesten für die Bischöfe von Valence seit der zweiten Hälfte des 11. Jahrhunderts. Diese Arbeit und die (wenigen) Regesten für die kleineren Stadtklöster in Valence (Saint Victor, Saint Vincent, Saint-Pierre du Bourglès-Valence, Saint-Félix-lès-Valence) sollen bis zu der für Anfang November geplanten Archivreise abgeschlossen sein, während der Frau Schilling an der Journée d'études in Valence zu Saint-Ruf teilnehmen wird. Dort möchte sie sich mit weiteren Kollegen über Saint-Ruf austauschen. Die Abschnitte zu den Zisterzienserklöstern der Diözese (Vernaison und Léonce) wurden bereits 2005/2006 samt historischen Einleitungen und Bibliographien fertiggestellt. Hier steht nur noch die Überprüfung der Archivalien aus, die bei ihrem Aufenthalt in Valence erfolgen soll. Das Lemma zu einem älteren Benediktinerkloster außerhalb der Stadt (Saou) mit nur wenigen Regesten hofft sie, mit den Stadtklöstern zusammen, noch vor der Archivreise wenigstens vorläufig abschließen zu können. Seit Mai/Juni arbeitet Frau Dagmar Hutter vom Erlanger Lehrstuhl ihr als Hilfskraft sehr kompetent zu. Sie hat bereits den Großteil des Manuskripts zu Grenoble Korrektur gelesen. Das Bistum Valence soll in diesem Winter abgeschlossen werden. Die Bearbeitung der Diözesen Die und Viviers soll im Anschluß geschehen; in einem Jahr wird noch einmal eine kürzere Archivreise in die Gegend notwendig sein. - IV/1-2: Erzdiözese Arles und Suffragane: Herr PD Dr. 
Stefan Weiß (Vechta) berichtet, daß er in den letzten Jahren seine Materialien stets aktualisiert habe, allerdings nicht dazu gekommen sei, die Arbeit über einen längeren Zeitraum konzentriert voranzubringen. - VIII/1-2: Erzdiözese Narbonne und Suffragane: Im Mittelpunkt der Forschungstätigkeit von Frau Dr. Ursula Vones-Liebenstein (Köln) stand die Abgrenzung der Kirchenprovinz Narbonne, vor allem im 11. und im 12. Jahrhundert, gegenüber der Kirchenprovinz Tarragona. In diesem Zusammenhang hielt sie auf der Tagung „Das begrenzte Papsttum“ in Lissabon (vgl. oben) einen Vortrag, in dem sie sich primär mit den Grenzbistümern Elne und Maguelonne beschäftigte. Die Arbeit an den Regesten des Kathedralkapitels Nîmes mußte wegen der Abfassung eines Aufsatzes im Rahmen des Netzwerkes „Zentrum und Peripherie“ einstweilen zurückstehen. Sie rechnet jedoch damit, die Regesten für dieses Kathedralkapitel und für die Abtei SaintBaudile im kommenden Jahr fertigzustellen. - Für die Indices zur „Gallia Pontificia" konnte Herr Hiestand in Göttingen einige offene Fragen klären und über Fernleihen einige weitere Lücken bei der Bestimmung der Ortsnamen schließen. In diesem Teil wird es bei einer Anzahl von Kirchen und Orten (ca. 20) vermutlich bei einem non liquet bleiben müssen. Der ganze Band soll zum Beginn des Jahres 2011 abgeschlossen werden.

Anglia Pontificia

Ein Bericht von Frau Prof. Dr. Julia Barrow (Nottingham) ist nicht eingegangen. - Der von Herrn Hiestand und Dr. Stefan Hirschmann (Köln) bearbeitete Band „Anglia Pontificia. Subsidia vol. I“ liegt nunmehr auch in einer elektronischen Fassung vor, so daß nach der Klärung einiger offener Fragen eine zügige drucktechnische Bearbeitung des Bandes möglich sein wird.

Iberia Pontificia (Leitung: Prof. Dr. Klaus Herbers, Erlangen)

Die Kooperation des Projektes mit spanischen und mit portugiesischen Wissenschaftlern und Wissenschaftlerinnen wurde fortgesetzt (zu den regelmäßigen Arbeitstreffen der Iberia-Mitarbeiter und der diesjährigen Konferenz vgl. auch oben).

Diözese León: Prof. Dr. Santiago Domínguez Sánchez (León) meldet weitere Fortschritte. Zur Zeit stellt er den Urkundenkatalog fertig, in den schon die Dokumente der delegierten Richter eingearbeitet wurden. Ferner sichtete er die Literatur aus der Frühen Neuzeit nach relevanten Informationen zu den Papstkontakten mit freilich nur spärlicher Ausbeute. Zur Zeit arbeitet er an der Abfassung der Einleitungen. - Erzdiözese Compostela: Die 
Arbeiten konnten im Berichtsjahr vom Sekretär geringfügig fortgeführt werden. - Suffragane: Prof. Dr. José Luis Martín Martín (Salamanca) hat die Bearbeitung der südlichen Suffraganbistümer von Compostela (Salamanca, Ciudad Rodrigo, Coria, Badajoz und Plasencia) fortgesetzt und sich dabei insbesondere um die Ermittlung von Deperdita bemüht. Mit diesem Ziel hat er begonnen, Kataloge, Inventare und sonstige Erwähnungen von Urkunden aus dem 16. bis zum 19. Jh. auszuwerten. Um die Diözesanzugehörigkeit von Empfängerinstitutionen genauer bestimmen zu können, hat der Bearbeiter sich eingehend mit dem Grenzverlauf zwischen den betreffenden Bistümern beschäftigt und konnte diese Untersuchungen in ein Referat auf der Lissaboner Tagung im Juli 2010 einfließen lassen. Die Erarbeitung des Regestenbandes profitierte von der Neubearbeitung der seinerzeit maßgeblich von Herrn Martín Martín erstellten Edition der Urkunden des Kathedral- und des Diözesanarchivs Salamanca (1. Aufl. 1977). - Erzdiözese Tarragona: Herr Prof. Dr. Ludwig Vones (Köln) konnte im Berichtszeitraum sein Projekt nur wenig voranbringen.

Portugalia Pontificia (Prof. Dr. Maria Cristina Almeida e Cunha, Porto) Ein Bericht lag nicht vor.

Zu den Diözesen Burgos und Ávila sowie zu den Aufzeichnungen aus spanischen Archiven und Bibliotheken vgl. den Arbeitsbericht unter „Die Forschungsvorhaben der Akademie, Papsturkunden des frühen und hohen Mittelalters".

\section{Scandinavia Pontificia}

Herr Prof. Dr. Anders Winroth (New Haven) meldet nur langsame Fortschritte bei der Erarbeitung seines Bandes. Im Berichtsjahr konnte er lediglich eine Miszelle zu JL. 7625 mit einem Wiederabdruck der litterae aus der sehr seltenen editio princeps von 1642 verfassen, die im Jahrgang 2010 des „Bulletin of Medieval Canon Law“ erscheinen wird. Des weiteren moderierte er im Juni eine Sektion bei der Konferenz „Die Ordnung der Kommunikation und die Kommunikation der Ordnungen im mittelalterlichen Europa. Zentralität: Papsttum und Orden im Europa des 12. und 13. Jahrhunderts" in der Villa Vigoni.

\section{Polonia Pontificia}

Vgl. dazu den Arbeitsbericht unter „Die Forschungsvorhaben der Akademie, Papsturkunden des frühen und hohen Mittelalters“. 
Bohemia-Moravia Pontificia

Vgl. dazu den Arbeitsbericht unter „Die Forschungsvorhaben der Akademie, Papsturkunden des frühen und hohen Mittelalters“.

Hungaria Pontificia (Leitung: Prof. Dr. Werner Maleczek, Wien)

Ein Bericht von Herrn Zsolt Hunyadi, PhD (Szeged) ist nicht eingegangen.

Dalmatia-Croatia Pontificia (Leitung: Prof. Dr. Werner Maleczek, Wien) Ein Bericht von Herrn Dr. Stjepan Razum (Zagreb) lag nicht vor.

Africa Pontificia

Ein Bericht von Herrn Prof. Dr. Peter Segl (Pfaffenhofen a. d. Ilm) ist nicht eingegangen.

Oriens Pontificius

I. Patriarchatus Hierosolymitanus et Antiochenus

Herr Hiestand konnte mit Hilfe von Frau Anne Kemmerich die bibliographischen Recherchen zum „Oriens“ weiterführen.

II. Domus fratrum Hospitalis et domus militiae Templi

Aufgrund von turnusgemäß zu übernehmenden Aufgaben in der akademischen Selbstverwaltung konnte Prof. Dr. Jochen Burgtorf (Fullerton, USA) das Projekt nur durch Literaturnachträge fördern. Im Berichtszeitraum publizierte er den Aufsatz "The Debate on the Trial of the Templars (1307-1314)“, ed. Jochen Burgtorf, Paul F. Crawford, and Helen J. Nicholson, editorial board: Malcolm Barber, Peter Edbury, Alan Forey, and Anthony Luttrell, Aldershot 2010.

Neubearbeitung des Jaffé

Vgl. dazu den Arbeitsbericht unter „Die Forschungsvorhaben der Akademie, Papsturkunden des frühen und hohen Mittelalters“.

Digitalisierung

Herrn Thorsten Schlauwitz oblag es, ein Digitalisierungsprojekt zu initiieren, das zu einem Antrag bei der DFG führen soll. Für diesen Zweck sind die 27 Urkundenphotos der Göttinger Arbeitsstelle zum Pontifikat Leos IX. (1049-1054) mit Hilfe des Göttinger Digitalisierungszentrums retrodigitalisiert und in die bereits vorhandene online-Datenbank eingebunden worden. Letztere wurde um entsprechende Funktionen ergänzt. $\mathrm{Zu}$ den genannten Digitalisaten sind die entsprechenden Regesten in die Datenbank eingefügt sowie Scans von entsprechenden Faksimiles, Editio- 
nen und weiteren Regestenwerken erstellt worden. Dies soll das gewünschte Endresultat - eine Vereinigung von Regest, Abbildung und Edition in einer Datenbank - illustrieren und somit einem Antrag dienen, der in Kooperation mit mehreren Institutionen bis Ende des Jahres eingereicht werden soll.

\section{Verschiedenes}

Vom 16. bis 18. Juni 2010 fand die vom Sekretär mitorganisierte Tagung „Die Ordnung der Kommunikation und die Kommunikation der Ordnungen im mittelalterlichen Europa. Zentralität: Papsttum und Orden im Europa des 12. und 13. Jahrhunderts" in der Villa Vigoni statt, wozu auch einige Mitarbeiter des Akademienprojektes und der Pius-Stiftung Vorträge beisteuerten. - Anfang September 2010 stellte Herr Herbers im Rahmen eines Vortrages das Gesamtunternehmen bei der Internationalen Konferenz „Pontes ad fontes“ in Brünn vor. - Mit Hilfe von Frau Anne Kemmerich konnte der Altsekretär die Sammlung von Drucken von Papsturkunden wieder um ca. 800 Stücke und 20 Legatenurkunden vermehren, die in die Göttinger Gesamtsammlung eingearbeitet werden.

K. Herbers

\section{Wörterbuch der Klassischen Arabischen Sprache}

Delegierter: Nagel

Kontaktadresse: Seminar für Arabistik, Papendiek 16, 37073 Göttingen, Tel.: 0551-39-4398, Fax: 0551-39-9898, arabsem@gwdg.de (Prof. Dr. Nagel)

Arbeitsbericht: Im März 2009 ist die 40. Lieferung des Wörterbuchs der Klassischen Arabischen Sprache erschienen. Damit ist der V. Band des Werkes abgeschlossen, der das Ende des Buchstabens Lām, das Literaturverzeichnis, einen Wortindex und einen Abriß der Geschichte der arabischen Lexikographie in Europa seit dem 17. Jahrhundert enthält. Alle fünf Bände sind in der Tübinger Redaktion hergestellt worden. Das Werk sollte in München in der Bayerischen Akademie der Wissenschaften mit dem Buchstaben Mìm fortgesetzt werden. Zu diesem Zweck sind dort seit 1975 umfangreiche Vorarbeiten geleistet worden. In ihrer Sitzung am 3. Februar 2006 hat die Philologisch-Historische Klasse der Akademie jedoch beschlossen, die Arbeiten bis auf weiteres einzustellen. 
Die Arabisten sind somit auch weiterhin auf materiell und methodisch unzureichende Hilfsmittel angewiesen. Es sind dies das „Lexicon ArabicoLatinum" von G. W. Freytag (Vol. I-IV, Halis Saxonum 1830-1837) und das unvollendete „Arabic-English Lexicon“"von E. W. Lane (Book I, Part 1-8, London and Edinburgh 1863-1893). Beide Werke reproduzieren lediglich das Material der einheimischen arabischen Lexikographen des Mittelalters, die mit ihrem normativen Ansatz die historische Entwicklung der Sprache ignorieren. In ihren Kompilationen sind weder die Wörter und Begriffe, die erst nach dem Sturz der Umaiyaden im Jahre 750 aufgekommen sind, noch die zahllosen griechischen, aramäischen und iranischen Fremdwörter erfaßt. Die Wortbedeutungen sind oft nur vage formuliert. Zwischen eigentlichem und metaphorischem Gebrauch wird kaum unterschieden, okkasionelle Bedeutungen stehen gleichberechtigt neben usuellen, und es gibt keine Angaben über das Niveau oder die Frequenz eines Wortes. Auch die Dependenzen der Verben sind kaum berücksichtigt. All diese Mängel haften auch den Wörterbüchern von Freytag und Lane an, so daß die Arabisten bei der Lektüre altarabischer und mittelalterlicher Texte oft im Stich gelassen sind, ganz zu schweigen davon, daß sprachwissenschaftliche Fragestellungen mit diesen alten Lexika nicht zu beantworten sind.

Unter diesen Umständen ist das vorläufige Ende der Arbeiten am WKAS ein schwerer Rückschlag für die Arabistik. Es bleibt zu hoffen, daß die lexikalischen Sammlungen in Tübingen (etwa 450.000 Belege auf Zetteln) und München (etwa 65.000 Belege) zu einem späteren Zeitpunkt doch noch redigiert und publiziert werden können.

T. Nagel

\author{
Ausschuss für musikwissenschaftliche Editionen \\ (Union der Akademien) \\ Delegierter: Heidrich
}

Deutsche Inschriften des Mittelalters und der frühen Neuzeit

(Interakademische Kommission)

Delegierter: Henkel

Deutsche Reichstagsakten, Ältere Reihe

Delegierter: Sellert 
Deutsches Museum München

(Vorstandsrat)

Delegierter: Kippenhahn

Göttingische Gelehrte Anzeigen

Redaktoren: Lehmann, Ringleben

Herausgabe des Thesaurus Linguae Latinae

(Interakademische Kommission)

Delegierter: Classen

Mittellateinisches Wörterbuch

Delegierter: Mölk

Patristik

(Kommission der Akademien der Wissenschaften in der Bundesrepublik

Deutschland)

Delegierter: Döpp

Zentraldirektion der Monumenta Germaniae Historica

Delegierter: Rexroth 